\title{
Prognostic role of secreted protein acidic and rich in cysteine in patients with solid tumors
}

Yongchen Ma, PhD, Hongbo Chen, PhD, Huiying Ma, MS, Zibao Yao, MD, Jianwen Hu, MS, $J u$ Ma, PhD, Xiaoqian Zhang, $P h D$, Guowei Chen, MD, PhD, Yucun Liu, MD, PhD.

\section{ABSTRACT}

$$
\begin{aligned}
& \text { الأهداف: لتحليل الوظائف غير المتجانسة للبروتين المفرز الحمضية }
\end{aligned}
$$

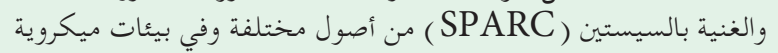

$$
\begin{aligned}
& \text { مختلفة للأورام بهدف تحديد أهميتها السريرية. }
\end{aligned}
$$

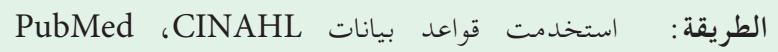
التع (Embase ، Cochrane ، Web of Science التي ركزت على آثار تعبير SPARC على تطور الورم الصلب والآلثار

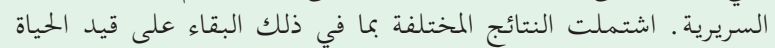

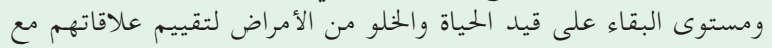

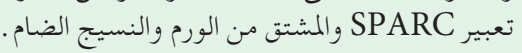

النتائج: سجلت دراسة التحليل التلوي الحالي 26 دراسة بما في ذلكٍٍ

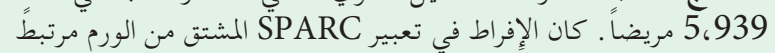

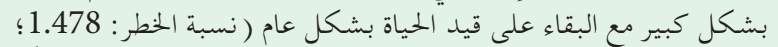

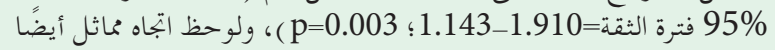

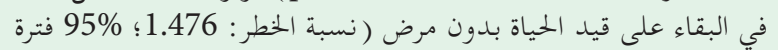

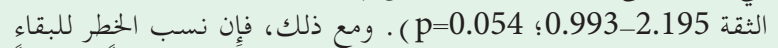

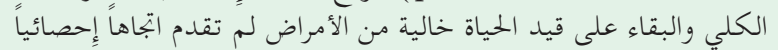

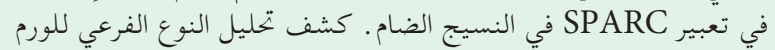

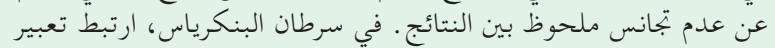
SPARC

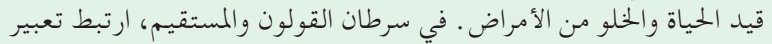
SPARC

الحناتمة: بالنسبة لمعظم الأورام الصلبة، قد يكون SPARC في الخلايا

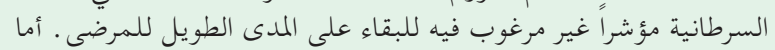

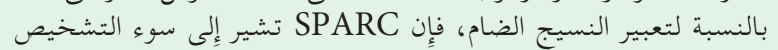

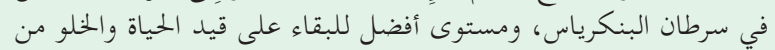

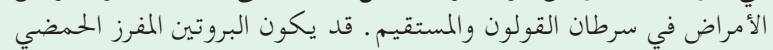

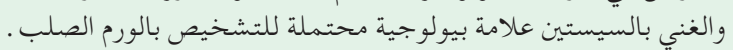

Objectives: To analyze the heterogeneous functions of secreted protein acidic and rich in cysteine (SPARC) from different origins and in different tumor microenvironments with the purpose of determining its clinical significance.

Methods: The PubMed, CINAHL, Cochrane, Web of Science and Embase databases were utilized. Studies that focused on the effects of SPARC expression on solid tumor progression and clinical implications were used. The different outcomes including overall survival and disease-free survival were analyzed to evaluate their relations with tumor- and stroma-derived SPARC expression.

Results: A total of 26 studies including 5,939 patients were enrolled in the present meta-analysis. Tumor-derived SPARC overexpression was significantly related with poor overall survival (hazard ratio: 1.478 ; $95 \%$ CI: 1.143 $1.910 ; p=0.003$ ), and a similar tendency was also observed in disease-free survival (hazard ratio: 1.476; 95\% CI: 0.993-2.195; $p=0.054$ ). However, the hazard ratios for overall survival and disease-free survival did not present a statistical trend in stromal SPARC overexpression. Tumor type subgroup analysis revealed marked heterogeneity among outcomes. In pancreatic cancer, SPARC overexpression in the stroma was significantly associated with poorer overall survival and disease-free survival. In colorectal cancer, SPARC overexpression in the stroma was associated with better disease-free survival.

Conclusion: For the majority of solid tumors, SPARC in cancer cells may be an unfavorable indicator for long-term survival for patients. As for stromal expression, SPARC indicates a poorer prognosis in pancreatic cancer, but a better disease-free survival in colorectal cancer. Secreted protein acidic and rich in cysteine might be a potential biomarker for solid tumor prognosis.

Saudi Med J 2019; Vol. 40 (8): 755-765

doi: $10.15537 /$ smj.2019.8.24379

From the Department of General Surgery (Ma Y, Yao, Hu, Ma J, Zhang, Chen G, Liu), Peking University First Hospital, from the Peking University School of Nursing (Chen H), Beijing, and from the Medical College, Qingdao University (Ma H), Shandong 266000, People's Republic of China.

Received 16th March 2019. Accepted 20th June 2019.

Address correspondence and reprint request to: Dr Guowei Chen, Department of General Surgery, Peking University First Hospital, Beijing, People's Republic of China. E-mail: guoweichen@263.net ORCID ID: https://orcid.org/0000-0002-7440-5639 
$\mathrm{T}$ he tumor microenvironment theory states that the survival and development of tumors are dependent on the whole tumor environment. ${ }^{1}$ Based on the modern cancer model, cancer-associated fibroblasts (CAFs), the predominant cellular components surrounding cancer cells, serve a crucial role in tumor proliferation, metastasis, drug resistance and other biological phenotypes. ${ }^{2}$ Secreted protein acidic and rich in cysteine (SPARC), mainly produced by fibroblasts, chondrocytes and osteoblasts, is a member of the matricellular family of proteins. ${ }^{3}$ Secreted protein acidic and rich in cysteine modulates interactions between cells and the extracellular matrix, participating in normal tissue remodeling and wound repair. ${ }^{4,5}$ Nowadays, emerging evidence has shown the participation of SPARC in various cancer progression. Though numerous studies have revealed that SPARC was expressed in different types of cancers, the exact function of SPARC is controversial. Some reports have shown that SPARC may contribute to cell adhesion, angiogenesis and proliferation, and the overexpression of SPARC was associated with tumor metastasis and poor prognosis in biliary tract, pancreatic, breast and non-small cell lung cancers. ${ }^{6-10}$ However, other studies have reported that SPARC served an inhibitory role in angiogenesis and tumor formation, resulting in favorable prognosis in gastric cancer and diffuse large B-cell lymphoma. ${ }^{11,12}$ Given that the different localization of SPARC also had different outcomes, Nakashima et $\mathrm{al}^{7}$ demonstrated that stromal SPARC expression was associated with several clinicopathological parameters and was indicative of a poor prognosis, while the tumoral SPARC expression within homologous niches failed to draw a significant conclusion. Therefore, the prognostic value of SPARC in the tumor microenvironment is still unclear, and the heterogeneity of the mechanisms among different solid tumors and different cellular locations also requires elucidation. Thus, in order to further clarify the role of SPARC in the malignant solid tumor microenvironment, the present study performed a meta-analysis to evaluate the expression level of SPARC in 9 different types of solid tumors and the expression differences between cancer and stromal cells, especially

Disclosure. Authors have no conflict of interests, and the work was not supported or funded by any drug company. The study was supported by a grant on Potential Therapeutic Approaches for Surgical Infection and the Underlying Mechanisms from the China Health \& Medical Development Foundation, Beijing, China.
CAFs. In addition, the present study systematically analyzed the heterogeneous functions of SPARC in different tumor micro-environments with the purpose of determining its clinical significance.

Methods. The review was reported in accordance with the Preferred Reporting Items for Systematic Reviews and Meta-Analyses (PRISMA) statement. ${ }^{13}$

Search strategy. Database and manual searches were undertaken to identify relevant articles written in the English language. A systematic search was conducted in May 2019 following PRISMA (Figure 1A) in 5 databases (PubMed, CINAHL, Cochrane, Web of Science and Embase). The search terms were as follows: ("osteonectin" OR "SPARC glycoprotein" OR "BM-40" OR "basement membrane tumor protein" OR "BM 40 protein" OR "SPARC" OR "secreted protein acidic and rich in cysteine") AND ("neoplasm" OR "neoplasia" OR "tumor" OR "tumour" OR "cancer" OR "carcinoma” OR "malignancy" OR "malignant" OR "cancerous" OR "carcinomatous" OR "cancroid"). The reference lists of any relevant systematic reviews found via the search were screened to identify potentially eligible additional primary studies. Appendices of the reference lists of the included studies were also undertaken.

Study selection. Articles were included if they were retrospective studies that focused on the clinical implications of SPARC expression on overall survival (OS) and disease-free survival (DFS). Additionally, the following criteria were also fulfilled: i) SPARC expression was evaluated by immunohistochemistry (IHC); ii) the location of SPARC was precisely described and the analysis was performed in cancer and stroma cells independently; and iii) targeted outcome data were either reported or were able to be estimated from the relevant published data. Articles were excluded if they fulfilled any of the following criteria: i) If they were literature reviews, abstracts, case reports or letters; ii) if enrolled patients received neoadjuvant treatment, such as chemotherapy or radiotherapy prior to surgery; iii) if studies were performed in vitro or without clinical data available for the present analysis; and iv) if studies were repeated or duplicated. Two authors conducted the search and independently reviewed the titles and abstracts according to the inclusion criteria. Two authors determined the selection of articles included in the sample. Disagreements about inclusion decisions were resolved by consensus and consultation with a third reviewer.

Data extraction. Two authors independently extracted the data from all of included papers into Excel spreadsheets. Discrepancies in the data were 


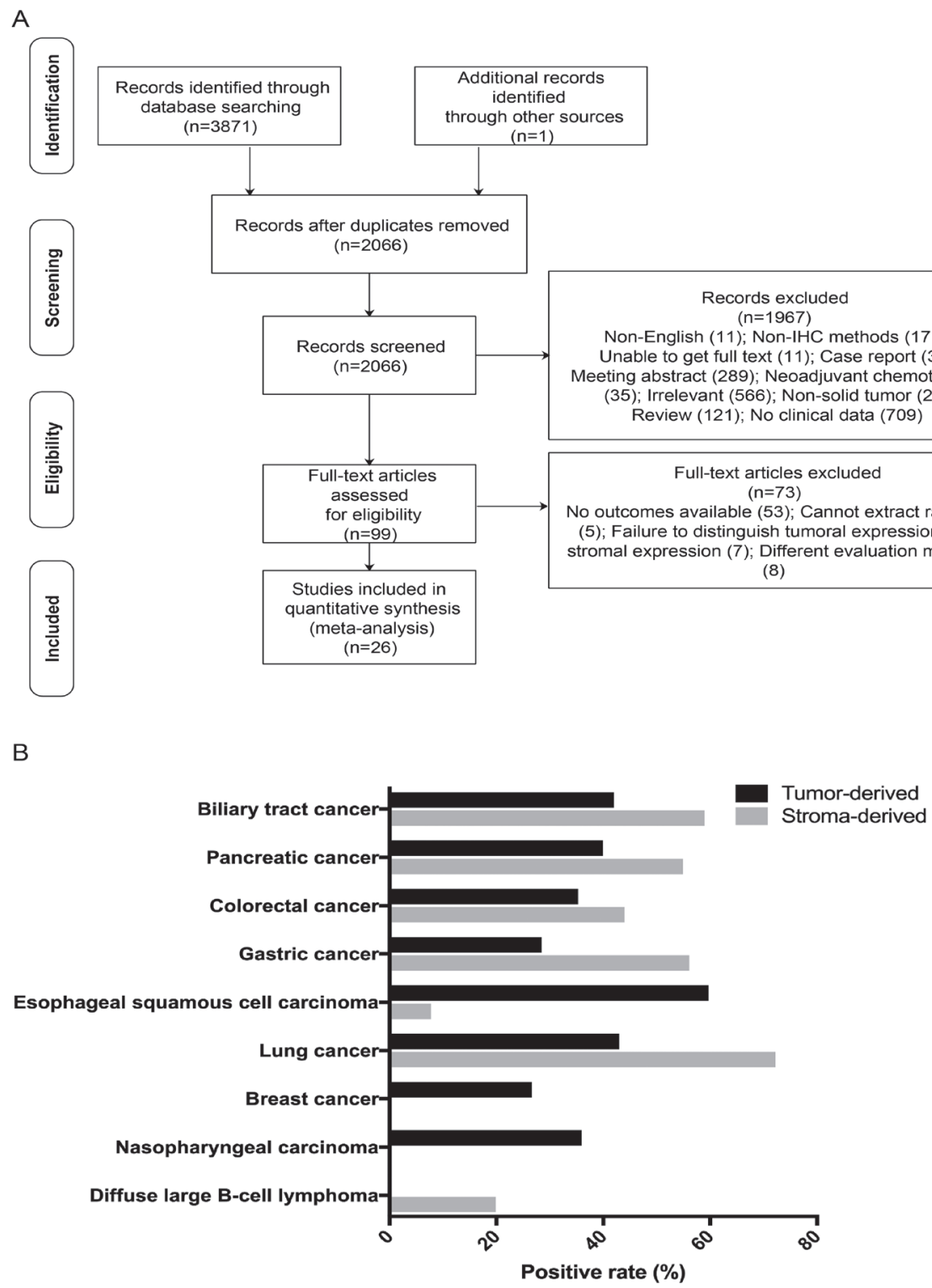

Figure 1 - Flow chart of the literature selection process and positive rates of secreted protein acidic and rich in cysteine (SPARC) in solid tumors. A) Flow chart of the literature search and selection process. B) Positive rates of SPARC in 9 types of solid tumors. The positive rates of SPARC varied among the 9 solid tumors, and in the same tumor type, the positive rates of SPARC expressed in cancer and stromal cells were also different. IHC - immunohistochemistry

resolved through consensus. The following information was collected from each study: first author, year of publication, country of origin, tumor type, number of enrolled patients, SPARC location, SPARC positive rate, SPARC antibody, cut-off value for overexpression, tumor size, invasion depth, lymph node metastasis, degree of differentiation, clinical stage, hazard ratios (HRs) with their $95 \%$ confidence intervals (95\% CIs) for OS and DFS. Methods described by Tierney et $\mathrm{al}^{14}$ were used to estimate the HRs and 95\% CIs if the survival data were not reported directly. Additionally, survival rates were extracted using the Engauge Digitizer for Mac version 10.11 based on Kaplan-Meier curves for the subsequent calculation.

Quality appraisal. Two reviewers independently evaluated the 26 articles using the Newcastle-Ottawa Scale (NOS), which has 8 items. ${ }^{15}$ This scale has been developed as the main evaluation method for non- 
randomized control studies and the scale for cohort studies was applied in the present analysis.

Statistical analysis. The meta-analysis was performed using STATA for Mac version 14.1 (StataCorp LP, College Station, TX, USA). The foremost outcomes for OS/DFS were measured based on the HR with 95\% CI. The heterogeneity of these studies was assessed by Q-test and $\mathrm{I}^{2}$-test. A random-effects model was applied when heterogeneity existed among studies $(p<0.05$ or $\mathrm{I}^{2}>50 \%$ ), otherwise a fixed-effects model was used. To explore the source of the heterogeneity of studies, subgroup analysis and meta regression were performed. The publication bias was evaluated by funnel plot and Egger's linear regression test, and $p<0.05$ indicated a significant publication bias among the enrolled studies. The stability of the results was confirmed by sensitivity analysis. A $p<0.05$ was considered to indicate statistically significant difference.

Results. Search results. The process of how studies were selected is presented in Figure 1A. The initial search identified 3,872 articles. Once the duplicates were removed and 2,066 titles and abstracts had been screened, 99 articles were identified for full-text review. Finally, 26 articles met the eligibility criteria. Studies were excluded due to the following reasons: i) No outcomes were available; ii) could not extract the raw data; iii) failure to distinguish tumoral expression from stromal expression; and iv) different evaluation methods.

Study characteristics. The basic characteristics of the enrolled studies were presented in Table 1. Briefly, the number of patients in each study ranged from 36 to 1,093 , and a total of 5,939 patients from 11 countries were included in the present meta-analysis. The studies focused on solid tumors, including gastric cancer, ${ }^{11,16-18}$ esophageal squamous cell carcinoma, ${ }^{19,20}$ pancreatic cancer, ${ }^{8,21-25}$ lung cancer, ${ }^{10,26,27}$ breast cancer, ${ }^{4,9,28}$ colorectal cancer, ${ }^{29-32}$ diffuse large B-cell lymphoma, ${ }^{12,33}$ biliary tract cancer, ${ }^{7}$ and nasopharyngeal carcinoma. ${ }^{34}$ The positive rates of SPARC varied among the 9 solid tumors, and in the same tumor type, the positive rates of SPARC expressed in cancer and stromal cells were also different (Figure 1B). The laboratory information and cut-off values for SPARC overexpression were presented in Appendix 1. The judgement of the cut-off varied depending on the methods they used, among which one study did not mention the evaluation of the cut-off value. ${ }^{4}$ For the convenience of the meta-analysis, some polytomous variables within studies were transformed into binary variables to calculate the OR or HR. Every eligible study gained a NOS score $>6$, indicating that all of the enrolled studies reached the high levels of methodological quality for meta-analysis (Appendix 2).

Overall survival. A total of 17 studies including 4,452 patients were used to analyze the association between SPARC overexpression in cancer cells and OS. Despite the high heterogeneity among the studies $\left(x^{2}=62.10, p<0.001, \mathrm{I}^{2}=74.2 \%\right)$, the pooled HR with multivariate analysis was 1.478 (95\% CI: 1.143-1.910; $p=0.003$; Figure $2 \mathrm{~A}$ ). Tumor type subgroup analysis showed that in gastrointestinal tumors, cancer-derived SPARC overexpression was indicative of a poorer OS (HR: 1.551; 95\% CI: 1.133-2.122; $p=0.006$; Figure 2B). And in respiratory tract tumors, SPARC was also negatively associated with OS (HR: 1.774; 95\% CI: $1.465-2.149 ; p<0.001$; Figure 2B). No publication bias was observed among the enrolled studies (PEgger=0.067).

As for stromal analysis, 15 studies with 3,244 patients were included and the random-effects model was applied $\left(x^{2}=69.63, p<0.001, \mathrm{I}^{2}=79.9 \%\right)$. However, no significant relation was observed (HR: $1.261 ; 95 \%$ CI: 0.908-1.751; $p=0.166$; Figure 3A). Additionally, the following subgroup analysis for precise tumor types revealed that, in pancreatic cancer, SPARC overexpression in the stroma was significantly associated with poorer OS (HR: 1.745; 95\% CI: 1.164-2.616; $p=0.007$; Figure $3 \mathrm{~B})$. The Egger's tests demonstrated the publication bias that existed among the enrolled studies (PEgger=0.009).

Disease-free survival. Data on the DFS of patients were reported by 5 studies in regard to cancer cells and 4 studies on stromal cells. The random-effects models were used for the two groups (cancer $x^{2}=14.57, p=0.006$, $\mathrm{I}^{2}=72.5 \%$; stromal $\left.x^{2}=25.33, \quad p<0.001, \quad \mathrm{I}^{2}=88.2 \%\right)$. As a result, SPARC overexpression in cancer cells was indicative of a higher risk of DFS, but the difference was not statistically significant (HR: 1.476; 95\% CI: 0.993-2.195; $p=0.054$; Figure 4A).

No statistically significant relation between SPARC overexpression in stromal cells and DFS was observed (HR: 0.903, 95\% CI: 0.465-1.754; $p=0.762$; Figure 4B). However, subsequent analysis of the tumor type subgroups revealed that in pancreatic cancer, SPARC overexpression in stromal cells was associated with poorer DFS, and in colorectal cancer, stroma-derived SPARC overexpression was associated with better DFS (pancreatic HR: 1.577, 95\% CI: 1.211-2.055; $p=0.001$; colorectal HR: 0.504, 95\% CI: 0.350-0.727; $p<0.001$ ). No publication bias was observed among the enrolled studies (cancer PEgger=0.201; stroma PEgger=0.309).

Meta regression. In order to influence the covariates, such as the design of the enrolled studies and 
Table 1 - Studies and clinical information of patients included in the meta-analysis.

\begin{tabular}{|c|c|c|c|c|c|c|c|c|c|c|}
\hline Study & Year & Country & Disease & $\begin{array}{l}\text { Case } \\
(\mathrm{n})\end{array}$ & $\begin{array}{l}\text { SPARC } \\
\text { location }\end{array}$ & Outcomes & $\begin{array}{c}\text { HR } \\
\text { obtainment }\end{array}$ & HR & $95 \% \mathrm{CI}$ & $\begin{array}{c}P- \\
\text { value }\end{array}$ \\
\hline Gao et $\mathrm{al}^{16}$ & 2015 & China & Gastric cancer & 78 & Cancer & OS & Estimated & 0.76 & $0.38-1.50$ & 0.661 \\
\hline Wu et $\mathrm{al}^{19}$ & 2017 & China & ESCC & 89 & Cancer & OS & Reported & 0.78 & $0.26-2.48$ & $>0.05$ \\
\hline \multirow[t]{4}{*}{ Sinn et $\mathrm{al}^{21}$} & 2014 & Germany & Pancreatic cancer & 160 & Cancer & OS & Estimated & 2.11 & $1.43-3.11$ & 0.011 \\
\hline & & & & & & DFS & Estimated & 1.57 & $1.15-2.14$ & 0.041 \\
\hline & & & & & Stroma & OS & Estimated & 1.37 & $0.94-2.01$ & 0.033 \\
\hline & & & & & & DFS & Estimated & 1.50 & $1.08-2.1$ & 0.005 \\
\hline Konrad et $\mathrm{al}^{17}$ & 2009 & Germany & Gastric cancer & 152 & Stroma & OS & Estimated & 0.72 & $0.25-2.09$ & 0.537 \\
\hline Michael et $\mathrm{al}^{26}$ & 2003 & Greece & NSCLC & 113 & Stroma & OS & Estimated & 1.47 & $0.74-2.93$ & 0.002 \\
\hline Youn et $\mathrm{al}^{28}$ & 1998 & Korea & Breast cancer & 253 & Cancer & OS & Reported & 0.60 & $0.33-1.08$ & 0.09 \\
\hline \multirow[t]{2}{*}{ Jeong et $\mathrm{al}^{29}$} & 2013 & Korea & Colorectal cancer & 332 & Cancer & OS & Reported & 2.10 & $1.05-4.19$ & 0.036 \\
\hline & & & & & Stroma & OS & Reported & 3.27 & $1.49-7.17$ & 0.003 \\
\hline Chris et $\mathrm{al}^{4}$ & 2004 & UK & Breast cancer & 566 & Cancer & OS & Reported & 6.88 & $1.75-27.04$ & 0.0057 \\
\hline \multirow[t]{2}{*}{ Xue et $\mathrm{al}^{20}$} & 2011 & China & ESCC & 116 & Cancer & OS & Estimated & 2.31 & $0.89-5.98$ & 0.944 \\
\hline & & & & & Stroma & OS & Reported & 1.09 & $0.57-2.08$ & 0.034 \\
\hline Masao et $\mathrm{al}^{11}$ & 2018 & Japan & Gastric cancer & 117 & Stroma & OS & Reported & 0.294 & $0.109-0.666$ & 0.002 \\
\hline \multirow[t]{2}{*}{ Ryuta et $\mathrm{al}^{22}$} & 2017 & Japan & Pancreatic cancer & 211 & Stroma & OS & Reported & 3.34 & $2.11-5.51$ & $<0.001$ \\
\hline & & & & & & DFS & Reported & 1.72 & $1.12-2.68$ & 0.013 \\
\hline Anamarija et al $^{12}$ & 2012 & USA & $\begin{array}{c}\text { Diffuse large B-cell } \\
\text { lymphoma }\end{array}$ & 125 & Cancer & OS & Reported & 0.48 & $0.26-0.91$ & 0.014 \\
\hline \multirow[t]{2}{*}{ Jeffrey et $\mathrm{al}^{23}$} & 2007 & USA & Pancreatic cancer & 299 & Cancer & OS & Reported & 1.02 & $0.73-1.42$ & 0.93 \\
\hline & & & & & Stroma & OS & Reported & 1.89 & $1.31-2.74$ & 0.001 \\
\hline \multirow[t]{2}{*}{ Shinsuke et $\mathrm{al}^{7}$} & 2014 & Japan & Biliary tract cancer & 110 & Cancer & OS & Reported & 1.61 & $0.99-2.63$ & 0.048 \\
\hline & & & & & Stroma & OS & Reported & 2.74 & $1.64-4.76$ & $<0.001$ \\
\hline \multirow[t]{2}{*}{ Neslihan et al ${ }^{10}$} & 2014 & Turkey & NSCLC & 84 & Cancer & OS & Reported & 1.97 & $1.2-3.21$ & 0.007 \\
\hline & & & & & & DFS & Reported & 1.67 & $1.05-2.66$ & 0.029 \\
\hline Jeung et $\mathrm{al}^{18}$ & 2011 & Korea & Gastric cancer & 80 & Cancer & OS & Reported & 2.01 & $1.18-3.4$ & 0.01 \\
\hline \multirow[t]{2}{*}{ Liang et $\mathrm{al}^{30}$} & 2010 & China & Colorectal cancer & 114 & Stroma & OS & Reported & 0.37 & $0.18-0.76$ & 0.006 \\
\hline & & & & & & DFS & Reported & 0.38 & $0.16-0.91$ & 0.03 \\
\hline Yu et $\mathrm{al}^{8}$ & 2017 & China & Pancreatic cancer & 73 & Cancer & OS & Reported & 2.37 & $1.20-4.67$ & 0.013 \\
\hline Chinmay et $\mathrm{al}^{24}$ & 2015 & Sweden & Pancreatic cancer & 88 & Stroma & OS & Reported & 2.12 & $1.19-3.98$ & 0.012 \\
\hline Wang et $\mathrm{al}^{34}$ & 2012 & China & Nasopharyngeal carcinoma & 1093 & Cancer & OS & Reported & 1.741 & $1.41-2.14$ & $<0.001$ \\
\hline \multirow[t]{4}{*}{ Liu et $\mathrm{al}^{31}$} & 2015 & China & Colorectal cancer & 740 & Cancer & OS & Reported & 0.805 & $0.52-1.26$ & 0.338 \\
\hline & & & & & & DFS & Reported & 0.770 & $0.53-1.12$ & 0.172 \\
\hline & & & & & Stroma & OS & Reported & 0.654 & $0.41-1.05$ & 0.028 \\
\hline & & & & & & DFS & Reported & 0.536 & $0.36-0.80$ & 0.002 \\
\hline Manuel et $\mathrm{al}^{25}$ & 2015 & Spain & Pancreatic cancer & 256 & Stroma & OS & Reported & 1.019 & $0.75-1.39$ & 0.903 \\
\hline Kazutoshi et $\mathrm{al}^{27}$ & 2016 & Japan & NSCLC & 200 & Stroma & OS & Reported & 1.58 & $0.65-3.85$ & 0.31 \\
\hline \multirow[t]{2}{*}{ Zhu et $\mathrm{al}^{9}$} & 2016 & China & Breast cancer & 211 & Cancer & OS & Reported & 1.90 & $1.14-3.16$ & 0.014 \\
\hline & & & & & & DFS & Reported & 1.73 & $1.10-2.73$ & 0.018 \\
\hline \multirow[t]{2}{*}{ Neslihan et $\mathrm{al}^{32}$} & 2016 & Turkey & Colorectal cancer & 43 & Cancer & OS & Reported & 7.01 & $2.06-23.85$ & 0.002 \\
\hline & & & & & & DFS & Reported & 4.04 & $1.19-13.70$ & 0.025 \\
\hline Paul et $\mathrm{al}^{33}$ & 2011 & Multinational & $\begin{array}{c}\text { Diffuse large B-cell } \\
\text { lymphoma }\end{array}$ & 236 & Stroma & OS & Estimated & 0.74 & $0.27-2.04$ & 0.006 \\
\hline
\end{tabular}

HR - hazard ratio, OS - overall survival, DFS - disease-free survival, ESCC - esophageal squamous cell carcinoma, NSCLC - non-small cell lung cancer

clinicopathological characteristics, towards the outcome OS, meta regression was perform in the present analysis. Year of publication, country, HR obtainment, tumor type and sample size were induced into the regression models. As a result, none of the covariates accounted for the sources of the heterogeneity in cancer cell group and stromal cell group (Appendix 3).

Funnel plot. The publication bias was evaluated by funnel plot when calculating all effect sizes
(Appendix 4A-4D). As a consequence, combined with the results of the Egger's linear regression test, the publication bias was observed when analyzing the association between SPARC overexpression in stromal cells and OS. And no significant publication bias was observed among other analysis.

Sensitivity analysis. In order to investigate the influence of individual study on the overall metaanalysis summary estimate, sensitivity analysis was also 
A

\begin{tabular}{|ll|}
\hline Study \\
ID
\end{tabular}

B

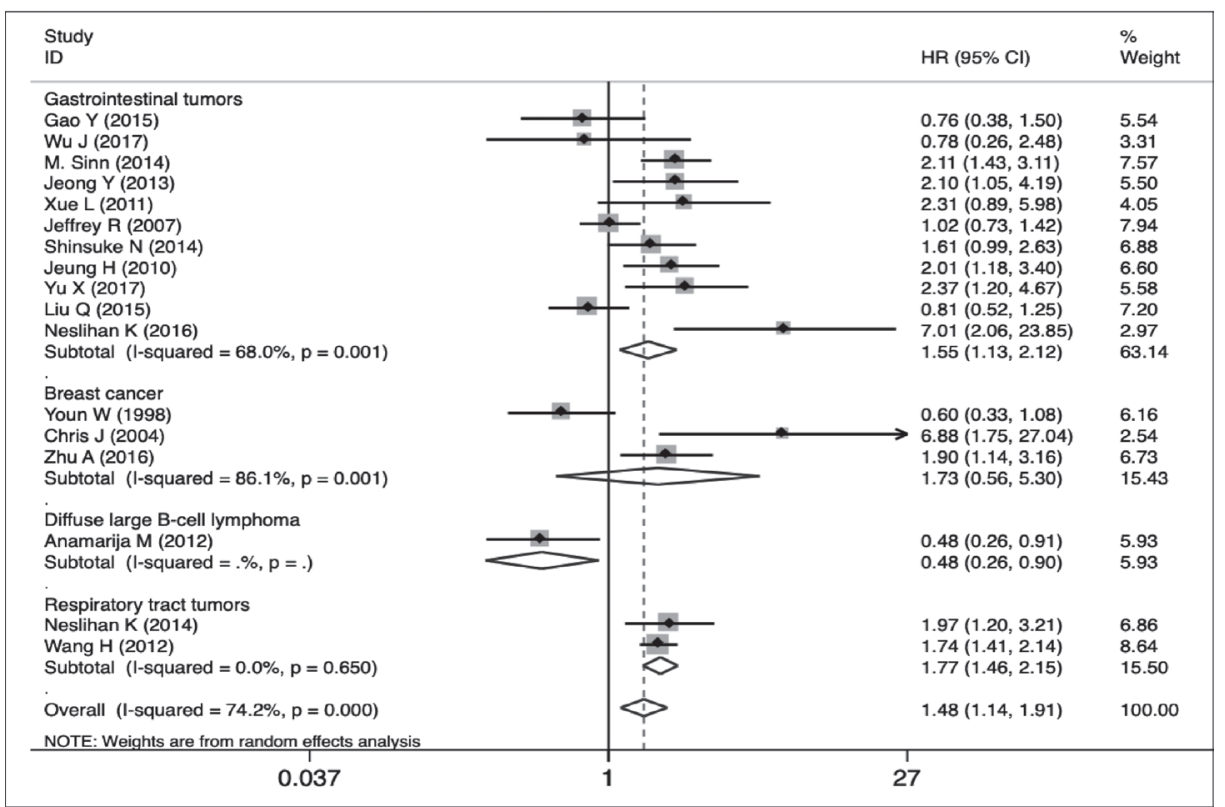

Figure 2 - Forest plot of the associations between tumoral secreted protein acidic and rich in cysteine (SPARC) over expression and overall survival (OS). A) OS in the cancer cell group. (B) Subgroup analysis of the tumor type for OS in the cancer cell group. HR - heart rate

performed among the evaluated outcomes with high heterogeneity using STATA 14.1. Outcomes including OS and DFS were assessed. In addition, no significant changes were detected between the previous and new effect sizes when one single study was deleted every time, which indicated that the results were relatively stable during the meta-analysis (Appendix 4E-4H).
Discussion. In the present meta-analysis, the HRs and $95 \%$ CIs were calculated for OS/DFS among enrolled studies to summarize the association between SPARC expression and long-term survival. However, the results changed with the translocation of SPARC expression. SPARC overexpression in cancer cells was significantly related with poor OS. In view of 

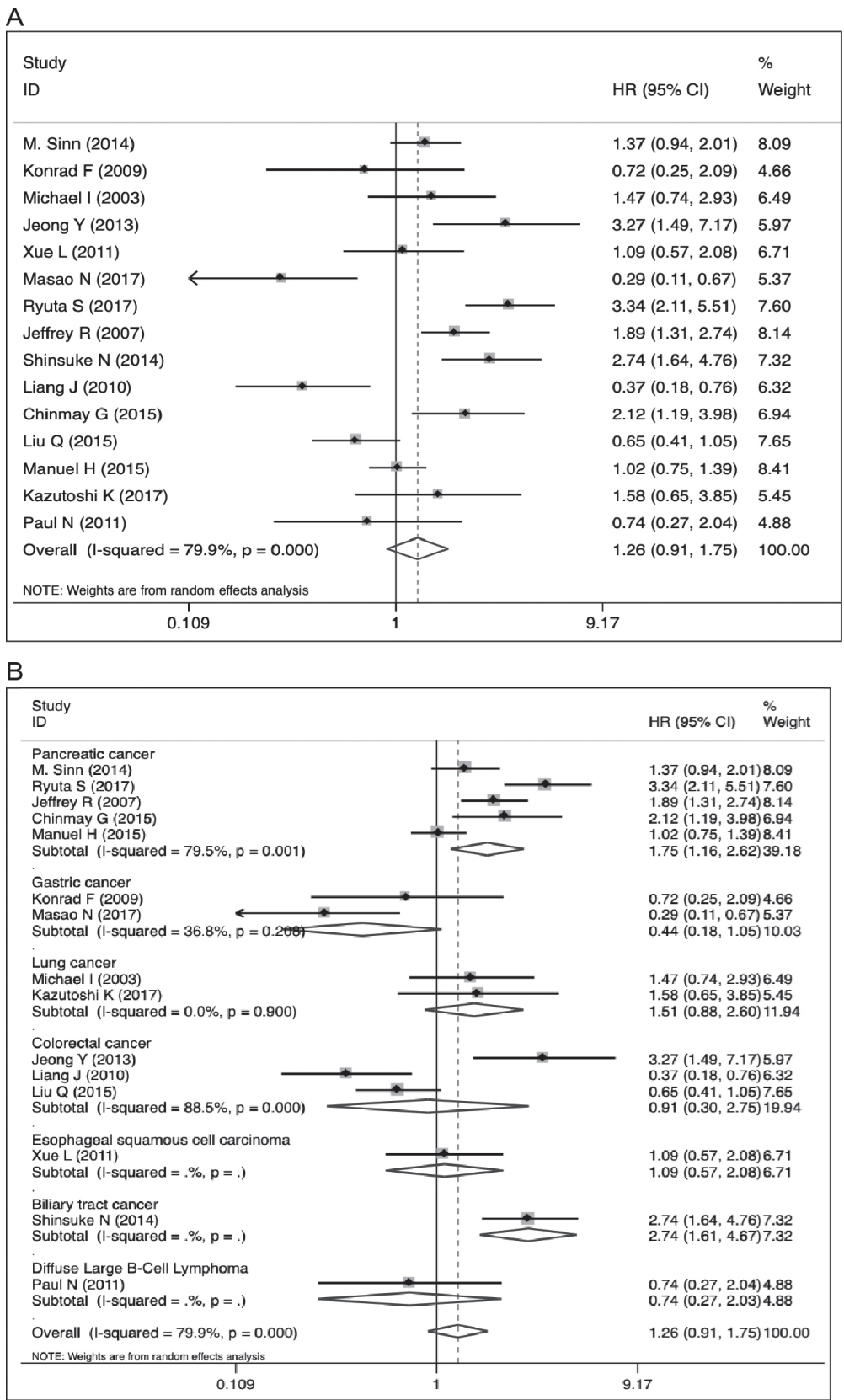

Figure 3 - Forest plot of the associations between stromal secreted protein acidic and rich in cysteine (SPARC) overexpression and overall survival (OS). A) OS in the stromal cell group. B) Subgroup analysis of the tumor type for OS in the stromal cell group. HR - heart rate

the heterogeneity, subgroup analysis was performed by stratifying the pooled data according to tumor types. In gastrointestinal and respiratory tract tumors, cancer-derived SPARC overexpression was indicative of a poorer OS. Similar results were also observed in the analysis for DFS, though the HR in the cancer cell group was not statistically significant. Additionally, the differences of sample sizes among the studies might be the source of the heterogeneity. For stromal analysis, the HR for OS and DFS did not present a statistical tendency in respect to SPARC overexpression. However, in pancreatic cancer, SPARC overexpression in the stroma was significantly associated with poor OS as well as DFS, and in colorectal cancer, SPARC overexpression was associated with better DFS, which indicated a marked tumor type heterogeneity in stroma-derived 


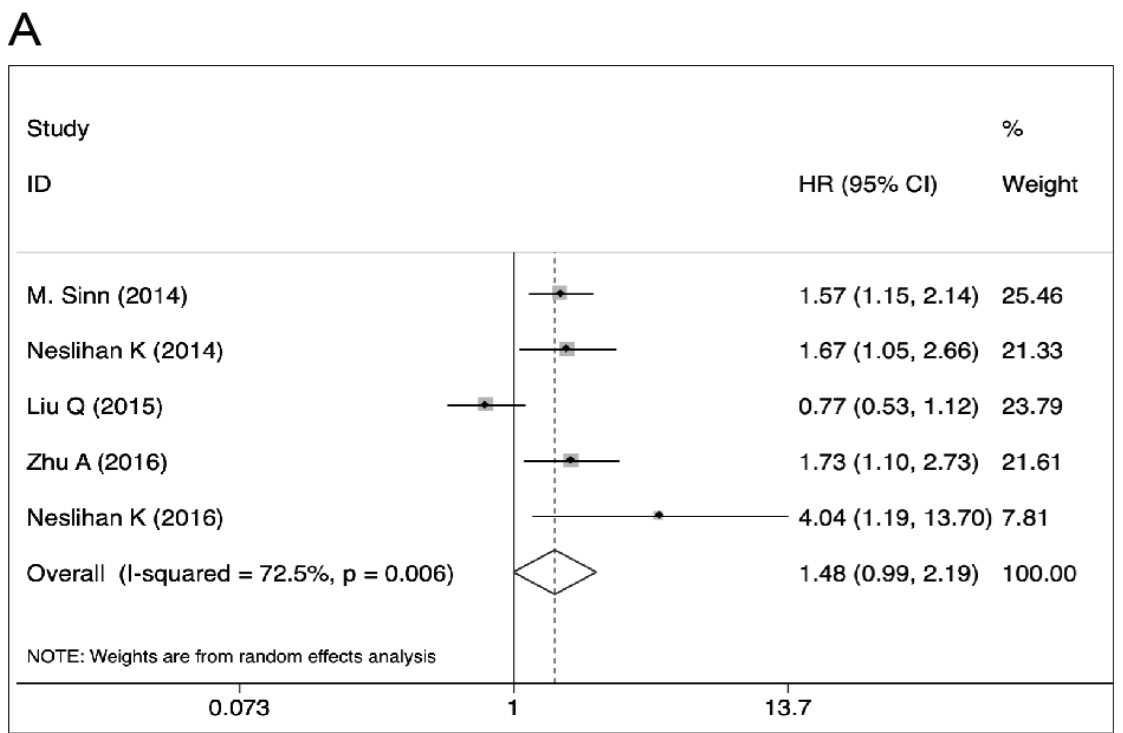

B

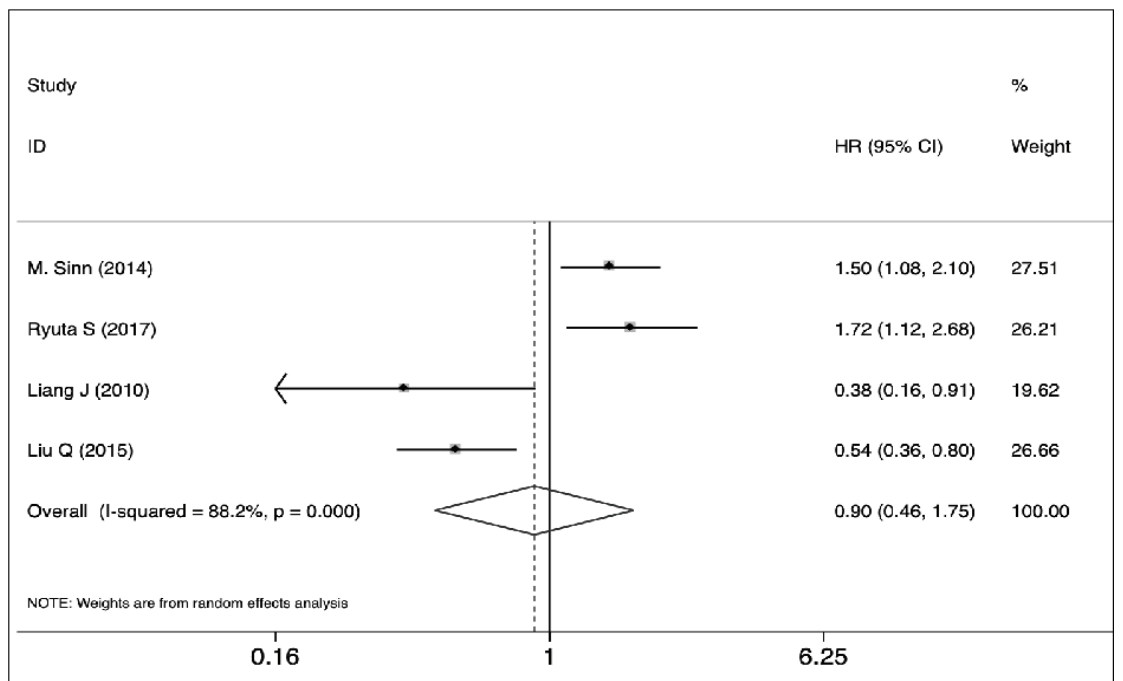

Figure 4 -Forest plot of the associations between secreted protein acidic and rich in cysteine (SPARC) overexpression and disease-free survival (DFS). A) DFS in the cancer cell group. B) DFS in the stromal cell group. HR - heart rate

SPARC. Although there was possible publication bias in the enrolled retrospective studies when analysis for OS in the stromal cell group was performed, the results were strengthened by the large sample size, and the source of the heterogeneity due to tumor types was also determined. Additionally, the sensitivity analysis also indicated a stable result. For the majority of solid tumors, SPARC overexpression in cancer cells may be an indicator for poor long-term survival, but the role in the stroma largely depends on the type of tumors.

Overall survival and DFS are effective indexes to assess the benefits or risks of the intervention in patients with cancer. Previous studies have reported various signaling pathways that SPARC may be involved in to affect the oncological outcomes, such as Urokinase-type plasminogen activator (uPA)-uPA receptor, phosphoinositide 3-kinase/protein kinase B, and mitogen activated protein kinase, contributing differently to patients' long-term survival. ${ }^{35,36}$ However, signaling pathways SPARC involved in among different tumor microenvironments may weighted diversely and lead to different outcomes, which partially accounts for the heterogeneity of SPARC in pancreatic cancer and colorectal cancer. Given its association with albumin 
binding, SPARC also participates in the response to adjuvant chemotherapy. Nab-paclitaxel, a first line drug for various types of cancers, can be trapped in the tumor stroma with the help of SPARC, and the stromal depletion effect enables cancer cells to have tumor-tumor and tumor-vessel communications. ${ }^{37,38}$ The interference in the therapeutic response may also make a difference in the survival rates.

The abilities of tumor migration and invasion largely depend on the strong viability of tumor cells and their frequentcrosstalk with the microenvironment. Oneof the most important mechanisms is epithelial-mesenchymal transition (EMT). Zhang et $\mathrm{al}^{139}$ demonstrated that in cancer metastasis mouse models, mice injected with SPARC-overexpressing gastric cancer cells had much fewer lung metastases than those administered the control cell injection. Previous studies have revealed that the inhibition of matrix metalloproteinase (MMP)-7, MMP-9, N-cadherin, Sp1 and extracellular signal-regulated kinase $1 / 2$ by SPARC may account for this phenomenon. Nevertheless, some studies have also reported that SPARC overexpression could interfere with collagen incorporation into basement membranes, which potentially destroyed the barrier function of basement membranes and facilitated invasive cells to breach the natural defense in situ. ${ }^{40}$ Thus, the roles of SPARC in the clinicopathological characteristics varied and we hypothesized that the effects of SPARC on tumor progression were systematically modulated and specifically weighted within different microenvironments.

During all events, divergent roles of SPARC in different types of tumor and different expression locations require further investigation. Additionally, the receptors and direct pathways that SPARC was involved in have not been discovered. Future studies should focus more on tracing the diverse paths of SPARC in tumor parenchyma as well as the stroma, and interactions within different tumor microenvironments.

The present study has some strengths and limitations. We comprehensively overviewed the expression level of SPARC in 9 types of solid tumors and the expression differences between cancer and stromal cells were evaluated in regard to clinicopathological characteristics and long-term survival. In order to minimize the heterogeneity as much as possible, the present study rigorously restricted the inclusion and exclusion criteria. In order to investigate the exact behaviors of SPARC from different cellular origins, only studies using the IHC method, which clearly located the expression positions in tumor tissues, were enrolled. In addition, studies with neoadjuvant therapy were excluded; some studies have reported that the expression level of SPARC could be altered when patients received chemotherapy. ${ }^{16}$ Additionally, sensitivity analysis was performed for all the results with high heterogeneity to verify the stability of the conclusions drawn.

Study limitations. Firstly, the studies mainly focused on pancreatic and colorectal cancer; other types of tumors such as nasopharyngeal carcinoma require more studies and larger sample sizes to support conclusions. Secondly, the enrolled studies were mainly retrospective, but not randomized controlled trials; although the present study tried to control confounding factors, the heterogeneity and publication bias inevitably existed among the eligible studies. The SPARC antibodies they applied, the method to define cut-off values and the ethnicity investigated may all be factors that influenced the homogeneity of the outcomes. Thirdly, some data on HRs were not provided in the original articles and thus, the present study estimated them from Kaplan-Meier curves with the help of an Engauge Digitizer, which led to inaccuracy to some degrees. Finally, only English articles were included, which may have caused the loss of some valuable studies published in other languages.

In conclusion, for the majority of solid tumors, SPARC overexpression in cancer cells may be an indicator of poor long-term survival, but their role in the stroma largely depends on tumor type. In pancreatic cancer, SPARC overexpression in the stroma is significantly associated with poor OS and DFS, and in colorectal cancer, stroma-derived SPARC overexpression is associated with a better DFS, which is indicative of the heterogeneity of SPARC in different tumor types and different expression locations. Secreted protein acidic and rich in cysteine may be a potential biomarker for solid tumor prognosis. More studies on certain tumors such as diffuse large B-cell lymphoma should be performed in the future to provide a comprehensive understanding of SPARC, and the detailed mechanisms also need further study to explain the heterogeneity in different tumor microenvironment.

Acknowledgment. The authors would like to acknowledge Mr. Xiaoyao Guo and Mr. Qiuhao Chen for language editing.

\section{References}

1. Hui LL, Chen Y. Tumor microenvironment: Sanctuary of the devil. Cancer Lett 2015; 368: 7-13.

2. Ma YC, Zhu J, Chen SW, Li TY, Ma J, Guo SH, et al. Activated gastric cancer-associated fibroblasts contribute to the malignant phenotype and 5-FU resistance via paracrine action in gastric cancer. Cancer Cell Int 2018; 18: 104.

3. Sasaki T, Miosge N, Timpl R. Immunochemical and tissue analysis of protease generated neoepitopes of BM-40 (osteonectin, SPARC) which are correlated to a higher affinity binding to collagens. Matrix Biol 1999; 18: 499-508. 
4. Jones C, Mackay A, Grigoriadis A, Cossu A, Reis-Filho JS, Fulford L, et al. Expression profiling of purified normal human luminal and myoepithelial breast cells: identification of novel prognostic markers for breast cancer. Cancer Res. 2004; 64: 3037-3045.

5. Wong SLI, Sukkar MB. The SPARC protein: an overview of its role in lung cancer and pulmonary fibrosis and its potential role in chronic airways disease. Br J Pharmacol 2017; 174: 3-14.

6. Said N. Roles of SPARC in urothelial carcinogenesis, progression and metastasis. Oncotarget 2016; 7: 67574-67585.

7. Nakashima S, Kobayashi S, Sakai D, Tomokuni A, Tomimaru Y, Hama N, et al. Prognostic impact of tumoral and/or peritumoral stromal SPARC expressions after surgery in patients with biliary tract cancer. J Surg Oncol 2014; 110: 1016-1022.

8. Yu XZ, Guo ZY, Di Y, Yang F, Ouyang Q, Fu DL, et al. The relationship between SPARC expression in primary tumor and metastatic lymph node of resected pancreatic cancer patients and patients' survival. Hepatobiliary Pancreat Dis Int 2017; 16: 104-109.

9. Zhu A, Yuan P, Du F, Hong R, Ding X, Shi X, et al. SPARC overexpression in primary tumors correlates with disease recurrence and overall survival in patients with triple-negative breast cancer. Oncotarget 2016; 7: 76628-76634.

10. Kurtul N, Eroglu C, Unal D, Tasdemir EA, Orhan O, Zararsiz $\mathrm{G}$, et al. Prognostic value of SPARC expression in unresectable NSCLC treated with concurrent chemoradiotherapy. Asian Pac J Cancer Prev 2014; 15: 8911-8916.

11. Nakajima M, Yoshino S, Kanekiyo S, Maeda N, Sakamoto K, Tsunedomi R, et al. High secreted protein acidic and rich in cysteine expression in peritumoral fibroblasts predicts better prognosis in patients with resectable gastric cancer. Oncol Lett 2018; 15: 803-812.

12. Perry AM, Cardesa-Salzmann TM, Meyer PN, Colomo L, Smith LM, Fu K, et al. A new biologic prognostic model based on immunohistochemistry predicts survival in patients with diffuse large B-cell lymphoma. Blood 2012; 120: 2290-2296.

13. Moher D, Liberati A, Tetzlaff J, Altman DG, Grp P. Preferred Reporting Items for Systematic Reviews and Meta-Analyses: The PRISMA Statement. J Clin Epidemiol 2009; 62: 1006-1012.

14. Tierney JF, Stewart LA, Ghersi D, Burdett S, Sydes MR. Practical methods for incorporating summary time-to-event data into meta-analysis. Trials 2007; 8.

15. Wells G, Shea B, O'Connell D, Peterson J, Welch V, Losos M. The Newcastle-Ottawa Scale (NOS) for assessing the quality if nonrandomized studies in meta-analyses. Available from URL: http://wwwohrica/programs/clinical_epidemiology/oxfordhtm.

16. Gao YY, Han RB, Wang X, Ge SH, Li HL, Deng T, et al. Change of SPARC expression after chemotherapy in gastric cancer. Cancer Biol Med 2015; 12: 33-40.

17. Franke K, Carl-McGrath S, Roehl F-W, Lendeckel U, Ebert MPA, Taenzer M, et al. Differential Expression of SPARC in Intestinal-type Gastric Cancer Correlates with Tumor Progression and Nodal Spread. Transl Oncol 2009; 2: 310-320.

18. Jeung HC, Rha SY, Im CK, Shin SJ, Ahn JB, Yang WI, et al. A randomized phase 2 study of docetaxel and S-1 versus docetaxel and cisplatin in advanced gastric cancer with an evaluation of SPARC expression for personalized therapy. Cancer. 2011; 117: 2050-2057.

19. Wu J, Zhang JR, Jiang XQ, Cao XG. Correlation between secreted protein acidic and rich in cysteine protein expression and the prognosis of postoperative patients exhibiting esophageal squamous cell carcinoma. Mol Med Report 2017; 16: 3401-3406.
20. Xue LY, Zou SM, Zheng S, Liu XY, Wen P, Yuan YL, et al. Expressions of the gamma2 chain of laminin-5 and secreted protein acidic and rich in cysteine in esophageal squamous cell carcinoma and their relation to prognosis. Chin J Cancer 2011; 30: 69-78.

21. Sinn M, Sinn BV, Striefler JK, Lindner JL, Stieler JM, Lohneis $\mathrm{P}$, et al. SPARC expression in resected pancreatic cancer patients treated with Gemcitabine: results from the CONKO-001 study. Ann Oncol 2017; 28: 2900.

22. Shintakuya R, Kondo N, Murakami Y, Uemura K, Nakagawa $\mathrm{N}$, Okano $\mathrm{K}$, et al. The high stromal SPARC expression is independently associated with poor survival of patients with resected pancreatic ductal adenocarcinoma treated with adjuvant gemcitabine in combination with S-1 or adjuvant gemcitabine alone. Pancreatology 2018; 18: 191-197.

23. Infante JR, Matsubayashi H, Sato N, Tonascia J, Klein AP, Riall TA, et al. Peritumoral fibroblast SPARC expression and patient outcome with resectable pancreatic adenocarcinoma. J Clin Oncol 2007; 25: 319-325.

24. Gundewar C, Sasor A, Hilmersson KS, Andersson R, Ansari D. The role of SPARC expression in pancreatic cancer progression and patient survival. Scand J Gastroenterol 2015; 50: $1170-1174$.

25. Hidalgo M, Plaza C, Musteanu M, Illei P, Brachmann CB, Heise C, et al. SPARC Expression Did Not Predict Efficacy of nab-Paclitaxel plus Gemcitabine or Gemcitabine Alone for Metastatic Pancreatic Cancer in an Exploratory Analysis of the Phase III MPACT Trial. Clin Cancer Res 2015; 21: 4811-4818.

26. Koukourakis MI, Giatromanolaki A, Brekken RA, Sivridis E, Gatter KC, Harris AL, et al. Enhanced expression of SPARC/ osteonectin in the tumor-associated stroma of non-small cell lung cancer is correlated with markers of hypoxia/acidity and with poor prognosis of patients. Cancer Res 2003; 63: 5376-5380.

27. Komiya K, Nakamura T, Nakashima C, Takahashi K, Umeguchi $\mathrm{H}$, Watanabe $\mathrm{N}$, et al. SPARC is a possible predictive marker for albumin-bound paclitaxel in non-small-cell lung cancer. Onco Targets Ther 2016; 9: 6663-6668.

28. Kim YW, Park YK, Lee J, Ko SW, Yang MH. Expression of osteopontin and osteonectin in breast cancer. J Korean Med Sci 1998; 13: 652-657.

29. Kim JY, Jeong D, Ahn TS, Kim HJ, Park DS, Park SY, et al. Expression of Secreted Protein Acidic and Rich in Cysteine in the Stroma of a Colorectal Carcinoma is Associated With Patient Prognosis. Ann Coloproctol 2013; 29: 93-99.

30. Liang JF, Wang HK, Xiao H, Li N, Cheng CX, Zhao YZ, et al. Relationship and prognostic significance of SPARC and VEGF protein expression in colon cancer. J Exp Clin Cancer Res 2010; 29: 71.

31. Liu QZ, Gao XH, Chang WJ, Wang HT, Wang H, Cao GW, et al. Secreted protein acidic and rich in cysteine expression in human colorectal cancer predicts postoperative prognosis. Eur Rev Med Pharmacol Sci 2015; 19: 1803-1811.

32. Kurtul N, Tasdemir EA, Unal D, Izmirli M, Eroglu C. SPARC: As a prognostic biomarker in rectal cancer patients treated with chemo-radiotherapy. Cancer Biomark 2017; 18: 459-466.

33. Meyer PN, Fu K, Greiner T, Smith L, Delabie J, Gascoyne $\mathrm{R}$, et al. The stromal cell marker SPARC predicts for survival in patients with diffuse large B-cell lymphoma treated with rituximab. Am J Clin Pathol 2011; 135: 54-61. 
34. Wang HY, Li YY, Shao Q, Hou JH, Wang F, Cai MB, et al. Secreted protein acidic and rich in cysteine (SPARC) is associated with nasopharyngeal carcinoma metastasis and poor prognosis. J Transl Med 2012; 10: 27.

35. Vaz J, Ansari D, Sasor A, Andersson R. SPARC: A Potential Prognostic and Therapeutic Target in Pancreatic Cancer. Pancreas 2015; 44: 1024-1035.

36. Shin M, Mizokami A, Kim J, Ofude M, Konaka H, Kadono Y, et al. Exogenous SPARC suppresses proliferation and migration of prostate cancer by interacting with integrin beta1. Prostate. 2013; 73: 1159-1170.

37. Garber K. Stromal Depletion Goes on Trial in Pancreatic Cancer. J Natl Cancer Inst 2010; 102: 448-450.
38. Gong L, Mao W, Chen Q, Jiang Y, Fan YJCC, Pharmacology. Analysis of SPARC and TUBB3 as predictors for prognosis in esophageal squamous cell carcinoma receiving nab-paclitaxel plus cisplatin neoadjuvant chemotherapy: a prospective study. Cancer Chemother Pharmacol 2019; 83: 639-647.

39. Zhang J, Wang P, Zhu J, Wang W, Yin J, Zhang C, et al. SPARC expression is negatively correlated with clinicopathological factors of gastric cancer and inhibits malignancy of gastric cancer cells. Oncol Rep 2014; 31: 2312-2320.

40. Morrissey MA, Jayadev R, Miley GR, Blebea CA, Chi Q, Ihara $S$, et al. SPARC Promotes Cell Invasion In Vivo by Decreasing Type IV Collagen Levels in the Basement Membrane. PLoS Genet 2016; 12: 2

\section{Case Reports}

Case reports will only be considered for unusual topics that add something new to the literature. All Case Reports should include at least one figure. Written informed consent for publication must accompany any photograph in which the subject can be identified. Figures should be submitted with a $300 \mathrm{dpi}$ resolution when submitting electronically. The abstract should be unstructured, and the introductory section should always include the objective and reason why the author is presenting this particular case. References should be up to date, preferably not exceeding 15 . 